\title{
Research on moving target tracking technology based on complex background
}

\author{
Xintao HUANG ${ }^{1, a}$, JunYANG ${ }^{1, b, ~}{ }^{*}$, Mengjiao WU $^{2, c}$ and CanZHAO ${ }^{3, d}$ \\ 1,2,3 School of Information Science and Engineering, Yunnan university, KunMing, China \\ a173471638@qq.com, bjunyang@ynu.edu.cn, ${ }^{c} 344786838 @ q q . c o m,{ }^{d} 781831625 @$ qq.com
}

Keywords: Moving object tracking ; meanshift ; Camshift

\begin{abstract}
With the needs of social development, video tracking based on the algorithm has gradually been in a hot research in the software.CamShift algorithm is a real-time tracking algorithm, using color histogram model get each image frame color projection, and according to previous frame tracking results to adjust the search window positions and sizes, resulting in the current image size and central location of the target.Primarily,this system is a moving object tracking detection system based on CamShift. the system can track moving objects on the indoor environment or to track the moving object in the video stream.
\end{abstract}

\section{INTRODUCTION}

Video tracking is one of core elements in the field of computer vision, the finding of it having very wide practical applications in many fields.With the upgrading of production equipment, more and more video capture device is applied to life. But the human working have two shortcomings in efficiency: one is inaccuracy of visible on the picture location of the small area. If the observation interval, or redundant in the target image, you will need to go to observe the target area again; the second is observation of fatigue. Observers working long time in the face of many Terminal window will appear observation of fatigue, resulting in missed the first images of important events.

Using computer algorithms to improve both of these issues, computer video processing can find and track at the same time, synchronously follow up according the picture content, and can trigger an events alert alarm when you preset the cases alert events.

The research of Surrey University, TLD[2], is considered to be the most efficient video object tracking algorithms. However, it is an efficient algorithm for high precision single target tracking.but not a universal solution.Most of the questions don't have a universal solution. The real situation being very complicated, there is no standard time-space lower bound. So research and development of video target tracking still has a very large extension of space.Continuously Adaptive Mean-SHIFT algorithm is a face tracking algorithm based on mean shift algorithm [3] proposed by Gary, Bradski in 1999.In fact, Camshift algorithm is an improved Mean shift algorithm. Except face tracking, it can still achieve better results in the other tracking problem.

\section{CAMSHIFT ALGORITHM FLOW AND IMPLEMENTATION}

\subsection{Introduction to shift Mean related concepts}

Mean Shift algorithm is a stepping clustering algorithm, which the original idea was proposed by Fukunaga et al. In 1975. And in 1995 Yizong Cheng defined a family of kernel function [3] and set a weight coefficient to make the shift Mean algorithm have a good performance for clustering high dimensional linear non separable data, which can be applied to wider areas.

The basic form of shift mean vector is described as follows:

Giving $n$ sample points $x_{i}, \mathrm{i}=1, \ldots, \mathrm{n}$, in a given $\mathrm{D}$ dimensional space $R^{d}$, the basic form of the Shift Mean vector at the point $x$ is defined as: 


$$
M_{h}(x) \equiv \frac{1}{k} \sum_{x_{i} \in S_{k}}\left(x_{i}-x\right)
$$

Where $S_{h}$ is a set of y points satisfying (1-2) relations in a high dimensional ball area of radius h. If there are n samples $x_{i}, \mathrm{k}$ is the number of the sample $x_{i}$ falling in the set $S_{h}$.

$$
S_{h}(x)=\left\{y:(y-x)^{T}(y-x) \leq h^{2}\right\}
$$

Where $\left(x_{i}-x\right)$ is the offset vector of the sample point $x_{i}$ with respect to the $x$ point, mean shift vector $M_{h}(x)(1-1)$ defined is a sum over all the offset vector of $\mathrm{k}$ sample points with relative to the point $x$ and then average.

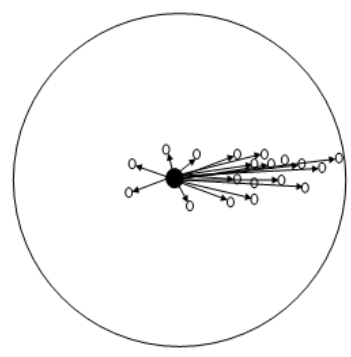

Fig. 1 Basic shift Mean vector and schematic

Intuitively, if the sample point $x_{i}$ is sampled from a probability density function,and since the non-zero probability density gradient is Pointing to the direction of maximum growth of probability density, On the average, the sample points in the region are more in the direction of the probability density gradient, corresponding shift Mean vector should point to the direction of the probability density gradient.

After introducing the kernel function in the basic shift Mean vector:

$$
M(x) \equiv \frac{\sum_{i=1}^{n} G_{H}\left(x_{i}-x\right) w\left(x_{i}\right)\left(x_{i}-x\right)}{\sum_{i=1}^{n} G_{H}\left(x_{i}-x\right) w\left(x_{i}\right)}
$$

Get (1-3) the extended shift Mean model in which:

$$
G_{H}\left(x_{i}-x\right)=|H|^{-1 / 2} G\left(|H|^{-1 / 2}\left(x_{i}-x\right)\right)
$$

$G(x)$ is a unit kernel function.

$H$ is a positive definite symmetric $d \times d$ matrix.

$w\left(x_{i}\right) \geq 0$, is assigned to the weight of the sampling point $x_{i}$.

If you meet all the sampling points $x_{i}$ :

a)

$$
w\left(x_{i}\right)=1
$$

b)

$$
G(x)= \begin{cases}1 & \text { if }\|x\|<1 \\ 0 & \text { if }\|x\| \geq 1\end{cases}
$$

All sampling points have the same weight, and the uniform kernel function is adopted, which can be reduced to (1-1) the basic form of 1-3. As shown in Figure 2, the shift Mean algorithm is always iterating in the direction of high interest signal density in the radius region. 


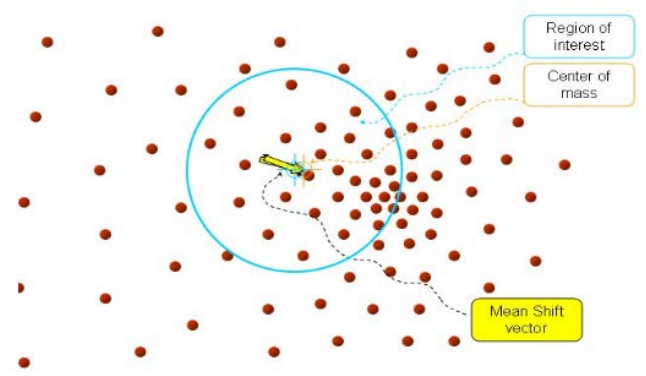

Fig. 2 schematic diagram of shift Mean algorithm

At the end of the 20th century, image processing is introduced into mean shift algorithm. usually Usually the probability density is defined as: Color approximation and the pixel coordinates are similar.

Until 2002 comaniciu et al the tracking problem approximation for a mean shift optimization problem [4], which make mean shift gradually develop into one of the highly efficient and mature theory systems in the Real-time tracking algorithms.

\subsection{Camshift Algorithm and Implementation}

based on the Mean shift, Camshift algorithm use the HSV color model to represent video images with a primary color features of the Hue channel. Secondly, adding the process of extracting the target feature frame by frame in the Mean shift step positioning tracking target location algorithm, The camshaft can adaptively obtain feature information of the Tracking target.

Camshift algorithm process can be described as follows[4]:

a) Read the first frame video image to convert RGB color mode to HSV color model, extract the Hue color component.

b) Ntervention selection determines the initial objectives and areas, and to initiating a search box, statistics Hue histogram of target area.(as show in figure3(a))

c) Centered at the center of the search box, according to the target area marked hue probability search radius of pixel matrix, convert the range image for reverse projection drawing. (as show in figure3(b))

d) To reverse projection with the Mean shift algorithm to calculate new centroid position of the search window, moving the center of the search box to the new centroid position, and update the size of the search box.

e) Gets the next frame. with the previous frame calculate the center and size of the search box and to initialize the current frame search box, and statistics Hue histogram of target area, go to (3) until the target leaving video images or the end of video image sequence.

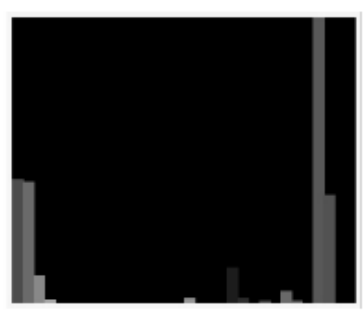

(a)

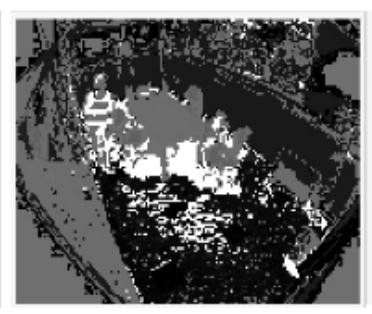

(b)

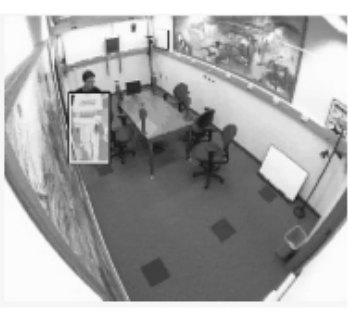

(c)

Fig. 3 Schematic diagram of Camshift tracking algorithm processing

Camshift algorithm can be implemented by OpenCv library based on C language, for the optimization of OpenCV good encapsulation for Mean shift, execution efficiency and the execution speed. 


\section{SUMMARY}

Camshift is a strongly adaptive, for complex background color videoand, and highly efficient algorithm, having a clear and easy to understand algorithm process and good extensibility.

Camshift does not require reading the entire video series, and can proceed frame-by-frame, save storage space, and realizes real-time tracking function, While the Mean shift algorithm may prove limited in iterations can always get a convergence of the results [9].

In numerous Camshift algorithm research findings, the characteristics of the target was not just limited to the hue parameter, and need hue and brightness both converging simultaneously, or add other features as special needs.Combined with different kernel functions and filter Camshift can be applied to different functional requirements, such as the 2010 Qian Yongqing's tracking algorithm based on Kalman prediction and Camshift, using Kalman filtering to improve selection accuracy of a search area [10] prior to the implementation of Mean shift algorithm.

So Camshift as a low resource consumption, high scalability of the algorithm, is currently the focus of video tracking algorithms, and provides a wide range of effective solutions for a wide range of low cost video tracking system.

\section{REFERENCES}

[1] Xue Muqing. Foreign media focus on large-scale installation of cameras [N]. China youth reference. August 10, 2011 (24).

[2] Kalal Z, Mikolajczyk K, Matas a. j. Tracking - Learning - Detection [J]. IEEE the TRANSACTIONS ON PATTERN ANALYSIS AND MACHINE INTELLIGENCE, 2010, 6 (1) : $1-14$.

[3] Yuan Guowu. Moving target detection and tracking algorithm of intelligent video surveillance research [D]. Yunnan university, 2012.

[4] Liu Yanli Tang Xianqi, yue-dong Chen. The movement target tracking algorithm based on improved Camshift application study [J]. Journal of anhui university of engineering, 2012, 27 (2) : 74-77.

[5] chinese-speaking, CAI zixing. MeanShift algorithm convergence analysis [J]. Journal of Software, 2007, 2 (18) : 205-212. 